\title{
Demethylation of the hTERT promoter in normal human gastric mucosal epithelial cells following $N$-methyl- $N^{\prime}$-nitro- $N$-nitrosoguanidine exposure
}

\author{
YONG-BO CHENG ${ }^{1}$, DIAN-CHUN FANG ${ }^{2}$, PING YAO $^{1}$, LI-PING GUO ${ }^{2}$, XIAO-YAN NING ${ }^{2}$ and LEI WANG $^{1}$ \\ ${ }^{1}$ Department of Gastroenterology, The First Affiliated Hospital of Xinjiang Medical University, Urumqi, Xinjiang 830054; \\ ${ }^{2}$ Department of Gastroenterology, Southwest Hospital, Third Military Medical University, Chongqing 400038, P.R. China
}

Received November 21, 2014; Accepted November 25, 2014

DOI: $10.3892 /$ br.2014.398

\begin{abstract}
N$-methyl- $N$ '-nitro- $N$-nitrosoguanidine (MNNG) is an alkylating agent that can induce gastric carcinoma. As a well-known human carcinogen, MNNG has been universally recognized as a methylating agent and is believed to act through methylation mechanism. In the present study, the epigenetic status of the human telomerase reverse transcriptase (hTERT) promoter was investigated in MNNG-treated normal human gastric mucosal epithelial cells. After $4 \mathrm{~h}$ exposure to $\mathrm{MNNG}$ at different concentrations, 6.8 and $68 \mu \mathrm{M}$, bisulfite sequencing polymerase chain reaction showed that five methylated cytosines outside the CpG dinucleotides in the 290-bp fragment from the hTERT promoter were demethylated and all the methylated cytosines in $\mathrm{CpG}$ dinucleotides remained intact. Furthermore, the epigenetic status of the target region following MNNG exposure was extremely similar to those of the BGC-823, SGC-7901 and MKN-28 lines; the three cell lines from human gastric adenocarcinoma. The result indicates that MNNG-induced demethylation in cytosines outside the $\mathrm{CpG}$ dinucleotides may be an early molecular lesion with the potential for impacting malignant transformation and a possible underlying carcinogenic mechanism of MNNG. Thus, it may provide another insight into the mechanisms of MNNG carcinogenesis.
\end{abstract}

\section{Introduction}

$N$-methyl- $N$ '-nitro- $N$-nitrosoguanidine (MNNG) is a well-known alkylating agent that can induce gastric carcinoma $(1,2)$. As a carcinogenic chemical, the mechanism of carcinogenesis induced by MNNG is not well understood.

Correspondence to: Professor Dian-Chun Fang, Department of Gastroenterology, Southwest Hospital, Third Military Medical University, 30 Gaotanyanzheng Road, Chongqing 400038, P.R. China E-mail: fangdianchun2012@163.com

Key words: $N$-methyl- $N$ '-nitro- $N$-nitrosoguanidine, carcinogenesis, demethylation, human telomerase reverse transcriptase, human gastric mucosal epithelial cell
Previous studies have focused on the methylation aspect $(1,3,4)$ and presently, MNNG has been recognized as a methylating agent universally $(5,6)$.

Thus far, MNNG-induced DNA epigenetic changes have been mostly reported in rats $(1,2,4-6)$, no relevant studies in humans found. Therefore, to improve the insight into the mechanism of human gastric carcinogenesis induced by MNNG, the epigenetic status of the human telomerase reverse transcriptase (hTERT) promoter, the rate-limiting subunit of telomerase whose activity is considered to be an early step in gastric carcinogenesis (7-9), was investigated in normal human gastric mucosal epithelial cells (nhGMECs) following MNNG exposure.

\section{Materials and methods}

Chemical. MNNG was purchased from Tokyo Chemical Industry Co.,Ltd., (Tokyo, Japan) dissolved in redistilled water at $25^{\circ} \mathrm{C}$ and formulated with a concentration of $0.1 \%$. Subsequently, the MNNG-containing water was confected into 6.8 and $68 \mu \mathrm{M}$ in Dulbecco's modified Eagle's medium (DMEM)-F12 medium (Gibco-BRL, Life Technologies, Carlsbad, CA, USA). The MNNG was freshly prepared for the experiment.

Cell culture. Following the approval by the The Third Military Medical University and the affiliated Southwest Hospital Ethical Committees and informed consent from the patient, nhGMECs were isolated from the specimen obtained by routine surgery with our previously developed method (10). The patient that provided the gastric sample was aged 42 years and underwent surgery for a gastric ulcer complicating perforation. The possibility of gastric cancer was excluded by postoperative pathology. BGC-823, SGC-7901 and MKN-28 cell lines were maintained in the laboratory and routinely cultured. All the cells were grown in DMEM-F12 medium supplemented with $10 \%$ fetal bovine serum (FBS) and without any antibiotics.

$M N N G$-treated cells. nhGMECs were cultured primarily for $48 \mathrm{~h}$. The supernatant was discarded and the cells were washed three times in warmed phosphate-buffered saline (PBS). Exposure for $4 \mathrm{~h}$ to MNNG at different concentrations 
TTTGAGAATTTGTAAAGAGAAATGACGGGTTTGTGTTAAGGAGTTTAAGTCGCGGGGAAGTGTTGTAGGGAGGTA Majority

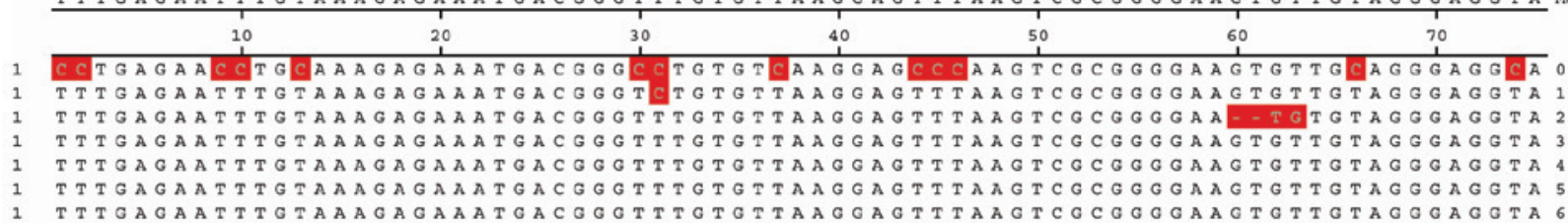

TTTCGGGAGGTTTCGCGTGTTCGTTTAGGGAGTAATGCGTTTTCGGGTTCGTTTTTAGTCGCGTTTACGCGTTTTMAOSITY

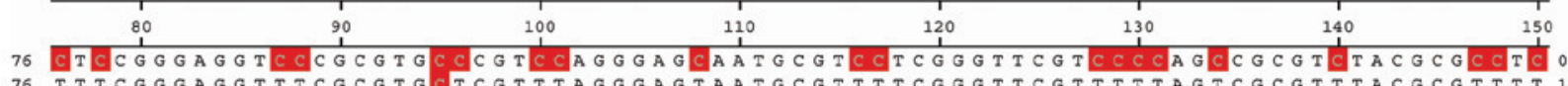

76 TTICGGGAGGTTICGCGTGCTCGTTTAGGGAGTAATGCGTTTTCGGGTTCGTTTTTAGTCGCGTTTACGCGTTTT

74 TTTCGGGAGGTTTCGCGTGTTCGTTTAGGGAGCAATGCGTTTTCGGGTTCGTTTTTAGTCGCGTTTACGCGTTTT2

76 TTTCGGGAGGTTTCGCGTGTTCGTTTAGGGAGTAATGCGTTTTCGGGTTCGTTTTTAGTCGCGTTTACGCGTTTT 3

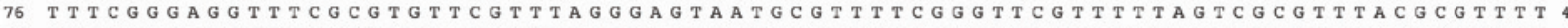

76 T T T C G G GAGGTTTCGCGTGTTCGTTTAGGGAGTAATGCGTTTTCGGGTTCGTTTTTAGTCGCGTTTACGCGTTTT

76 T T T CGGGAGG TTTCGCGTGTTCGTTTAGGGAGTAATGCGTTTTCGGGTTCGTTTTAGTCGCGTTTACGCGCTTT

CGTTTTTTTTTTT-ACGTTCGGTATTCGTGGTGTTCGGAGTTCGACGTTTCGCGTTCGGATTTGGAGGTAGTTTTMajority

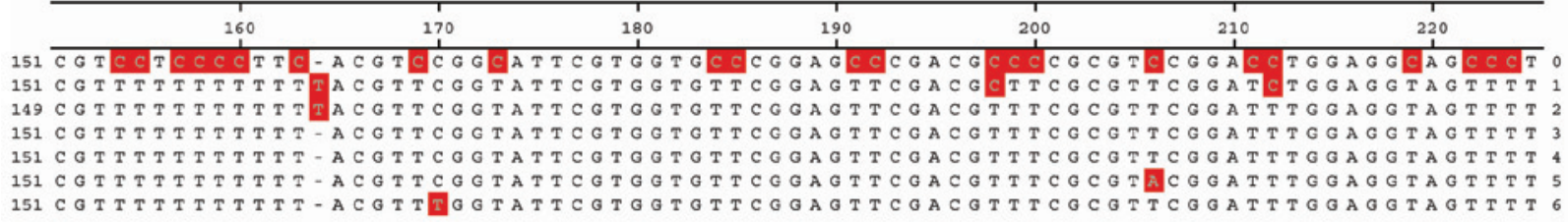

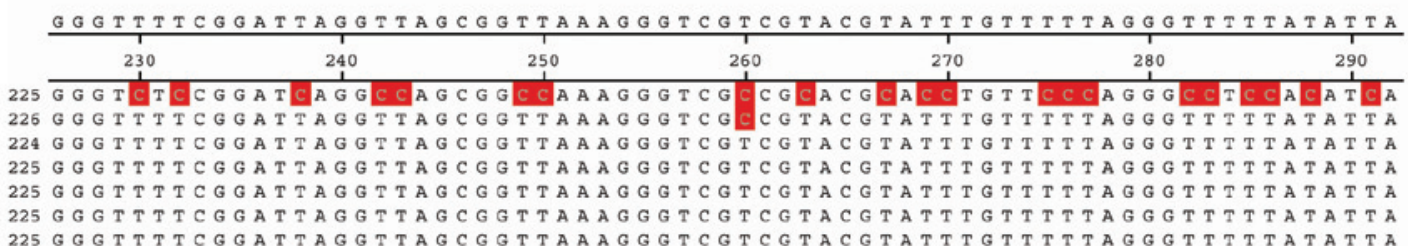

Majority

Figure 1. DNA sequencing result of the promoter region of human telomerase reverse transcriptase following bisulfite treatment. 0, Fragment in GenBank accession no. AF098956 sequence; 1, normal human gastric mucosal epithelial cells (nhGMECs) without $N$-methyl- $N$ '-nitro- $N$-nitrosoguanidine (MNNG) treatment; 2, nhGMECs treated with $6.8 \mu \mathrm{M}$ MNNG; 3, nhGMECs treated with $68 \mu \mathrm{M}$ MNNG; 4, BGC-823 cells; 5, SGC-7901 cells; 6, MKN-28 cells. Sequenator, ABI Prism 3730.

of 6.8 and $68 \mu \mathrm{M}$ was carried out. The cells were cultured in DMEM-F12 medium without MNNG and FBS as a negative control. Following treatment, the cells were washed five times with warmed PBS to remove any residual MNNG and reincubated in fresh DMEM-F12 medium supplemented with FBS. At intervals of $48 \mathrm{~h}$ ( $\leq 100 \mathrm{~h}$ post-isolation), the cells were harvested and studies were performed as described below.

hTERT promoter methylation assay. DNA were isolated from nhGMECs that were treated with and without MNNG, BGC-823, SGC-7901 and MKN-28 cells using E.Z.N.A. ${ }^{\circledR}$ SQ DNA kit (Omega Bio-Tek Inc., Norcross, GA, USA). The hTERT promoter methylation status was assessed with bisulfite sequencing polymerase chain reaction (PCR) using the EZ DNA methylation kit (Zymo Research, Irvine, CA, USA) as follows: Genomic DNA was modified by sodium bisulfite. A segment of 290 basepairs (bp) of the hTERT promoter (GeneBank accession no. AF097365) was amplified by PCR with primers: Forward, 5'-TTTGAGAATTTGTAAAGAGAAATG-3' and reverse, 5'-AATATAAAAACCCTAAAAACAAATAC-3'; under 32 cycles of $94^{\circ} \mathrm{C}$ for $50 \mathrm{sec}, 53^{\circ} \mathrm{C}$ for $50 \mathrm{sec}$ and $72^{\circ} \mathrm{C}$ for $1 \mathrm{~min}$, followed by $8 \mathrm{~min}$ at $72^{\circ} \mathrm{C}$. Sequencing of all the PCR products following cloning was performed by Sangon, Shanghai, China using the ABI Prism 3730 sequenator. All the sequence comparisons were carried out with the DNA Star software (DNASTAR Inc., Madison, WI, USA).

\section{Results}

MNNG treatment and the methylation status. The nhGMECs were dissociated and cultured. DNA were isolated from nhGMECs that were treated with and without MNNG, BGC-823, SGC-7901 and MKN-28 cells. Bisulfite treatment of genomic DNA can cause unmethylated cytosines to be completely converted into uracil, which are detected as thymine following PCR amplification, whereas methylated cytosines remain unchanged. The six sequences of the bisulfite-treated DNA were compared to those published in GenBank respectively. All 5'-CpG-3' dinucleotides were found to be methylated, with only five cytosines at positions 31, 95, 198, 212 and 260 outside the CpG dinucleotides methylated in the 290-bp fragment from the hTERT promoter region of the nhGMECs. Following 6.8 and $68 \mu \mathrm{M}$ MNNG exposure, all the methylated 5'-CpG-3' dinucleotides remained intact, the five cytosines were all demethylated and the methylation status of the target region was extremely similar to those of the BGC-823, SGC-7901 and MKN-28 lines (Fig. 1). The demethylated cytosines were at position 31, 95, 198, 212 and 260, which corresponded respectively to the $-716,-652$, $-550,-536$ and -488 positions, relative to the ATG translation initiation site (GeneBank accession no. AF097365).

\section{Discussion}

Primary cultured nhGMECs have a clear similarity with their corresponding cells in vivo and are taken as the ideal tool for 
gastric pathological studies. The methylation status of the hTERT promoter plays a role in hTERT expression and subsequent telomerase activation. The process may be an early step in gastric carcinogenesis (9). The aim of the present study, using nhGMECs, was to elucidate the mechanism of human gastric carcinogenesis induced by MNNG from the point of the epigenetic state of the hTERT promoter. As a carcinogenic agent, MNNG has been reported to produce DNA methylation and is usually recognized as a methylating agent (1,4-6). The present study observed that the epigenetic change of MNNG-treated nhGMECs was in agreement with those in previous studies. By contrast, it showed a selective demethylation in the 290-bp fragment from the hTERT promoter region. Furthermore, the demethylation of cytosine occured at position cytosine outside the $\mathrm{CpG}$ dinucleotides, and the methylated cytosine in the $\mathrm{CpG}$ dinucleotides remained intact. Notably, such epigenetic status of the target region following MNNG exposure was extremely similar to those of BGC-823, SGC-7901 and MKN-28 lines; the three cell lines from human gastric adenocarcinoma. This experiment could not be repeated due to the limited gastric specimens from similar patients. Therefore, the mechanism of human gastric carcinogenesis induced by MNNG may be correlated with demethylation in the hTERT promoter region or another undetected region. Previous studies on the mechanism of carcinogenesis have focused on more methylation and less demethylation. In neoplasia, demethylation of the genome as a whole occurs in vivo (11). Additionally, increasing the incidence of cancer during aging is accompanied by decreasing DNA methylation $(12,13)$, although there is controversy regarding this (8). From another perspective, the promoter of the hTERT gene becomes methylated during the development of some but not all tumors (14). All these indicate a possible role for demethylation in carcinogenesis. The epigenetic state of cytosine outside the $\mathrm{CpG}$ dinucleotides may be involved in the carcinogenesis. By computational prediction it has been estimated that 29,000 CpG-rich regions are distributed in the human genome (15); therefore, the majority of studies on gene methylation focused on cytosine in the $\mathrm{CpG}$ dinucleotides and revealed that the $\mathrm{CpG}$ islands within gene promoters generally become methylated during human carcinogenesis. As cytosine in the $\mathrm{CpG}$ dinucleotides, cytosine outside the $\mathrm{CpG}$ dinucleotides are also distributed in the promoter and first exon of genes and contain putative binding motifs, such as myeloid-specific zinc finger protein $2(16,17)$. Their epigenetic state may also affect the binding of transcription factors (16) and possibly result in carcinogenesis. Therefore, the epigenetic status of cytosine outside the $\mathrm{CpG}$ dinucleotides requires further study.

In the present study, to prevent interference MNNG was dissolved in redistilled water and not dimethylsulphoxide and was freshly prepared for the experiment. nhGMECs were all primary, not passage cells $(8,12)$ and were cultured in DMEM-F12 medium without any antibiotics. In general, alkylating agents, such as MNNG, produce increased $\mathrm{G} \rightarrow \mathrm{A}$, not $\mathrm{C} \rightarrow \mathrm{T}$, transition mutations. All five $\mathrm{C} \rightarrow \mathrm{T}$ outside the $\mathrm{CpG}$ dinucleotides are not considered to result from the mutagenic effect of MNNG (5).

In conclusion, a selective demethylation in the hTERT promoter in nhGMECs was observed following exposure to different MNNG doses in vitro. Demethylation in cytosine outside the $\mathrm{CpG}$ dinucleotides may be an early molecular lesion with the potential for impacting malignant transformation and a possible underlying carcinogenic mechanism of MNNG. Thus, it may provide another insight into the mechanisms of MNNG carcinogenesis.

\section{Acknowledgements}

The present study was supported by The National Natural Science Foundation of China (grant no. 30270609).

\section{References}

1. Gombar CT and Magee PN: DNA-methylation by nitrosocimetidine and N-methyl-N'-nitro-N-nitrosoguanidine in the intact rat. Chem Biol Interact 40: 149-157, 1982.

2. Manikandan P, Murugan RS, Priyadarsini RV, Vinothini G and Nagini S: Eugenol induces apoptosis and inhibits invasion and angiogenesis in a rat model of gastric carcinogenesis induced by MNNG. Life Sci 86: 936-941, 2010.

3. Haerlin R, Sussmuth R and Lingens F: Mechanism of mutagenesis by N-methyl-N'-nitro-N-nitrosoguanidine(MNNG)v.methylation of DNA by N-trideuteriomethyl-N'-nitro-N-nitrosoguanidine (D -MNNG). FEBS Lett 9: 175-176, 1970.

4. Bhalla A, Sachdeva $G$ and Bamezai R: T-cell receptor-gamma rearrangement and c-myb methylation in MNNG exposed Bloom syndrome B-lymphoblastoid cells. Cancer Lett 126: 1-6, 1998.

5. Schroering AG and Williams KJ: Rapid induction of chromatin-associated DNA mismatch repair proteins after MNNG treatment. DNA Repair (Amst) 7: 951-969, 2008.

6. Tomicic MT, Christmann M, Fabian K and Kaina B: Apaf-1 deficient mouse fibroblasts are resistant to $\mathrm{MNNG}$ and MMS-induced apoptotic death without attenuation of Bcl-2 decline. Toxicol Appl Pharmacol 207 (Suppl 2): 117-122, 2005.

7. Sabah M, Cummins R, Leader M and Kay E: Expression of human telomerase reverse transcriptase in gastrointestinal stromal tumors occurs preferentially in malignant neoplasms. Hum Pathol 35: 1231-1235, 2004.

8. Neumeister P, Albanese C, Balent B, Greally J and Pestell RG: Senescence and epigenetic dysregulation in cancer. Int J Biochem Cell Biol 34: 1475-1490, 2002.

9. Gulmann C, Lantuejoul S, Grace A, Leader M, Patchett S and Kay E: Telomerase activity in proximal and distal gastric neoplastic and preneoplastic lesions using immunohistochemical detection of hTERT. Dig Liver Dis 37: 439-445, 2005.

10. Cheng YB, Fang DC, Guo LP, Wang ZQ, Luo YH and Zhou JC: A convenient primary culture of human gastric epithelial cells. Chin J Gastroenterol 12: 31-35, 2007.

11. Issa JP: Aging, DNA methylation and cancer. Crit Rev Oncol Hematol 32: 31-43, 1999.

12. Suzuki T, Fujii M and Ayusawa D: Demethylation of classical satellite 2 and 3 DNA with chromosomal instability in senescent human fibroblasts. Exp Gerontol 37: 1005-1014, 2002.

13. Liu L, Lai S, Andrews LG and Tollefsbol TO: Genetic and epigenetic modulation of telomerase activity in development and disease. Gene 340: 1-10, 2004.

14. Devereux TR, Horikawa I, Anna CH, Annab LA, Afshari CA and Barrett JC: DNA methylation analysis of the promoter region of the human telomerase reverse transcriptase (hTERT) gene. Cancer Res 59: 6087-6090, 1999.

15. Santourlidis S, Wernet P, Ghanjati F, Graffmann N, Springer J, Kriegs C, Zhao X, Brands J, Arauzo-Bravo MJ, Neves R, et al: Unrestricted somatic stem cells (USSC) from human umbilical cord blood display uncommitted epigenetic signatures of the major stem cell pluripotency genes. Stem Cell Res 6: 60-69, 2011.

16. Poole JC, Andrews LG and Tollefsbol TO: Activity, function, and gene regulation of the catalytic subunit of telomerase (hTERT). Gene 269: 1-12, 2001.

17. Fujimoto K, Kyo S, Takakura M, Kanaya T, Kitagawa Y, Itoh H, Takahashi $\mathbf{M}$ and Inoue $\mathrm{M}$ : Identification and characterization of negative regulatory elements of the human telomerase catalytic subunit (hTERT) gene promoter: possible role of MZF-2 in transcriptional repression of hTERT. Nucleic Acids Res 28: 2557-2562, 2000. 